\title{
Automatic Assessment of Expanded Disability Status Scale (EDSS) in Multiple Sclerosis Using a Decision Tree
}

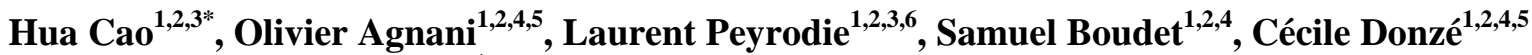 \\ ${ }^{1}$ Université Lille Nord de France, Lille, France \\ ${ }^{2}$ UCLille, Lille, France \\ ${ }^{3}$ Unité de Traitement des Signaux Biomédicaux, Hautes Etudes d’Ingénieur, Lille, France \\ ${ }^{4}$ Faculté Libre de Médecine, Groupe Hospitalier de l’Institut Catholique Lillois, Lille, France \\ ${ }^{5}$ Service de Médecine Physique et de Rééducation Fonctionnelle, Hôpital de Saint-Philibert, Lomme, France \\ ${ }^{6}$ Laboratoire d'Automatique et Génie Informatique et Signal, Université de Lille 1, Lille, France \\ Email: *hua.cao@hei.fr
}

Received 2013

\begin{abstract}
The expanded disability status scale (EDSS) is frequently used to classify the patients with multiple sclerosis (MS). We presented in this paper a novel method to automatically assess the EDSS score from posturologic data (center of pressure signals) using a decision tree. Two groups of participants (one for learning and the other for test) with EDSS ranging from 0 to 4.5 performed our balance experiment with eyes closed. Two linear measures (the length and the surface) and twelve non-linear measures (the recurrence rate, the Shannon entropy, the averaged diagonal line length and the trapping time for the position, the instantaneous velocity and the instantaneous acceleration of the center of pressure respectively) were calculated for all the participants. Several decision trees were constructed with learning data and tested with test data. By comparing clinical and estimated EDSS scores in the test group, we selected one decision tree with five measures which revealed a $75 \%$ of agreement. The results have signified that our tree model is able to automatically assess the EDSS scores and that it is possible to distinguish the EDSS scores by using linear and non-linear postural sway measures.
\end{abstract}

Keywords: Multiple Sclerosis (MS); Expanded Disability Status Scale (EDSS); Center of Pressure (COP); Recurrence Quantification Analysis (RQA); Decision Tree

\section{Introduction}

The expanded disability status scale (EDSS), proposed by Kurtzke [1], is frequently used to classify and standardize the patients with multiple sclerosis (MS). The EDSS score ranges from 0 (normal neurological examination) to 10 (death from MS) in 0.5 unit increments. There are eight functional systems (FS) involved to calculate the score: pyramidal, cerebellar, brain stem, sensory, bowel and bladder, cerebral, visual and other. EDSS is an ordinal measure and differences between the scale steps are not homogeneous. To overcome the variability between neurologists involved in examining the patients, at least a difference of 1.0 EDSS unit has been needed for defining a significant clinical change [2].

People with MS often present with poor balance which can be quantified by the force platform posturography, i.e. the trajectory of center of pressure (COP). During quiet standing on a force platform, two types of measures are usually derived from the posturologic data (COP sig-

*Corresponding author. nal) for evaluating postural performance: linear and nonlinear measures of postural sway. Linear measures, such as mean and standard deviation of sway amplitude, can mask the temporal variability of postural sway, while non-linear measures, such as measures of recurrence quantification analysis (RQA), can provide dynamical features of COP oscillations of a given time series.

Some studies on balance control in MS patients $[3,4]$ have demonstrated that sway (postural disorders) is significantly greater in the MS group than in controls. In our previous papers [5,6], we observed a significant correlation between the EDSS and the posturologic data. Therefore, the purpose of this paper was to present a novel method of automatic assessment of EDSS scores from measures of postural sway using a decision tree.

\section{Materials and Methods}

\subsection{Experiments}

There were two groups of participants (between 25 and 68 years of age) in our study: one of 118 participants (89 
patients and 29 healthy subjects) as the learning group and the other of 20 patients as the test group. This study was realized in the Hôpital de Saint-Philibert, Lomme, France. Neurologists established their clinical EDSS scores ranging from 0 to 4.5 . Nobody had orthopedic problems. This balance analysis technique is based on measurement of the COP's sway in a standing subject with a recording time of 51.2 seconds and a sampling frequency of $40 \mathrm{~Hz}$. The participant stood upright on a Satel platform (Figure 1) with bare feet and with his arms by the side. They were asked to stand as still as possible with the eyes closed during the record.

\subsection{Measures of Postural Sway}

\subsubsection{Linear Measures}

Two linear measures were computed for all the participants: the length $(\mathrm{L})$ representing the total length of the COP path, and the surface (S) corresponding to the surface area of the ellipse that enclosed $90 \%$ of the COP points computed by principal component analysis (PCA).

\subsubsection{Non-Linear Measures}

As posturogram reflects the movement of the COP, four non-linear RQA measures [7] were respectively calculated for COP's position (P), instantaneous velocity (V) and acceleration (A) (Table 1) of each participant.

Recurrence rate $(R e c)$ : which is expressed as the density of recurrence points in the recurrence plot (RP)

$$
\operatorname{Rec}=\frac{1}{N^{2}} \sum_{i, j=1}^{N} R(i, j),
$$

where $N$ represents the number of points on the COP's space trajectory and $R(i, j)$ represents the value of the point $(i, j)$ in the RP.

Shannon entropy (Ent): which represents the probability of finding a diagonal of a given length

$$
\text { En } t=-\sum_{l=l_{\min }}^{N} p(l) \ln p(l),
$$

where $l_{\min }$ is the minimal diagonal length, and $p(l)$ is the probability of a diagonal line of exactly length $l$ in the RP. This probability can be estimated from the frequency

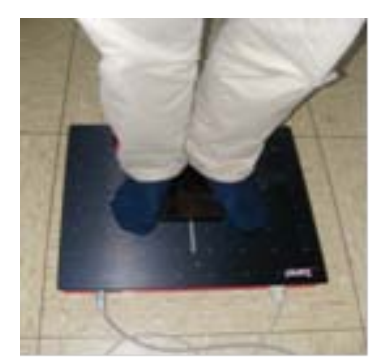

(a)

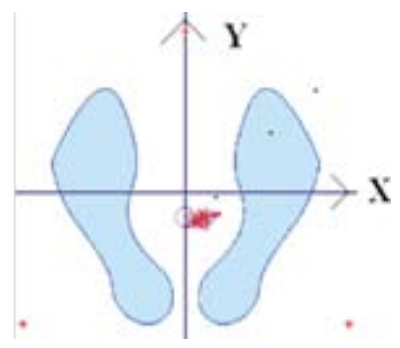

(b)
Figure 1. Satel force platform (a) and measurement of the COP (b).
Table 1. Formula for calculating the COP's instantaneous velocity and acceleration.

\begin{tabular}{cl}
\hline \multicolumn{1}{c}{ Parameter } & \multicolumn{1}{c}{ Formula } \\
\hline Instantaneous velocity (V) $\quad V(i)=\sqrt{(V x(i))^{2}+(V y(i))^{2}}$ \\
& $V x(i)=(\mathbb{X}(i+1)-X(i-1)) /(2 * \Delta t)$ \\
& $V y(i)=(Y(i+1)-Y(i-1)) /(2 * \Delta t)$ \\
& $A(i)=\sqrt{(A x(i))^{2}+(A y(i))^{2}}$ \\
Instantaneous acceleration (A) & $A x(i)=(\operatorname{Kk}(i+1)-V x(i)) /(\Delta t)$ \\
& $A y(i)=(V y(i+1)-V y(i)) /(\Delta t)$ \\
\hline
\end{tabular}

$\Delta t$ : sample duration equal to $1 / 40 \mathrm{~s}$.

distribution $P(l)$ with

$$
p(l)=\frac{P(l)}{\sum_{l=l_{\min }}^{N} P(l)} .
$$

Averaged diagonal line length $(L L)$ : which measures the average length of the diagonal lines

$$
L L=\frac{\sum_{l=l_{\min }}^{N} P(l)}{\sum_{l=l_{\min }}^{N} P(l)},
$$

which is related with the predictability time of the dynamical system.

Trapping time (TT): which quantifies the average length of the vertical lines

$$
T T=\frac{\sum_{v=v_{\min }}^{N} v P(v)}{\sum_{v=v_{\min }}^{N} P(v)}
$$

where $P(v)$ is the frequency distribution of the lengths $v$ of the vertical lines, which have at least a length of $v_{\min }$.

For calculating these measures, the time delay was set to $1 / 40 \mathrm{~s}$ and the embedding dimension was 1 . The minimal diagonal length was set to 2 samples. The radius thresholds for identify recurrence were set to $15 \mathrm{~mm}$ for the position, $39 \mathrm{~mm} / \mathrm{s}$ for the velocity and $360 \mathrm{~mm} / \mathrm{s}^{2}$ for the acceleration, because the maximal correlation coefficients between EDSS and recurrence measures were obtained with these radius for the learning group.

\subsection{Data Analysis}

Decision tree analysis [8] was performed in order to select the most important measures (among 14 measures mentioned previously) for assessing EDSS scores. We constructed our decision tree with all the possible combinations of no more than 5 measures of the learning group, because a combination of too many measures would increase the complexity of the tree. In order to evaluate each constructed tree, the percentage of agreement (\%Agreement) with an error of \pm 0.5 EDSS steps allowed was calculated by comparing estimated and clinical EDSS scores of the learning group. If its \%Agree- 
ment was greater than $80 \%$, we accepted this tree. Otherwise, we rejected it. All the retained trees were then tested with the data of the test group by computing their respective \%Agreement. The tree with the best agreement was finally selected as the most performant decision tree for the assessment of the EDSS score.

In addition, EDSS scores of the test group were also estimated by 2 second-order polynomial regression models (the surface of the ellipse and the recurrence rate of the COP's position) presented in our previous paper [6] in order to compare these two methods. These two models are described as follows:

$$
\text { EDSS }=0.095 *(\log (S))^{2}-0.12 * \log (S)-1.6,
$$

and

$$
\begin{aligned}
\text { EDSS } & =-0.94 *(\log (\operatorname{Rec} P))^{2} \\
& -3.9 * \log (\operatorname{Rec} P)+0.23,
\end{aligned}
$$

where $\mathrm{S}$ represents the surface of the ellipse and RecP is the recurrence rate of the position.

\section{Results}

One combination of five measures was finally selected by the decision tree analysis. The measures were S, L, Rec of the COP's position (RecP), TT of the velocity (TTV) and of the acceleration (TTA) respectively. The classification of the tree was described as follows ( $S$ in $\mathrm{mm}^{2}, \mathrm{~L}$ in $\left.\mathrm{mm}\right)$ :

1 if $\mathrm{S}<910.53$ then node 2 else node 3;

2 if TTA $<15.01$ then node 4 else node 5;

3 if $\mathrm{S}<2149.38$ then node 6 else node 7 ;

4 if TTA $<6.65$ then node 8 else node 9;

5 if TTA $<49.77$ then node 10 else node 11 ;

6 if $\operatorname{ReP}<0.58$ then node 12 else node 13;

7 if $\mathrm{S}<3180.81$ then node 14 else node 15;

8 class = 'EDSS 3';

9 if $\mathrm{L}<1536.59$ then node 16 else node 17 ;

10 if $\mathrm{S}<614.63$ then node 18 else node 19;

11 class = 'EDSS 0';

12 if TTA $<6.65$ then node 20 else node 21;

13 class = 'EDSS 2.5';

14 if $\mathrm{L}<2865.80$ then node 22 else node 23;

15 if $\mathrm{L}<3052.50$ then node 24 else node 25;

16 class = 'EDSS 2';

17 class = 'EDSS 2.5';

18 if $\mathrm{S}<131.54$ then node 26 else node 27 ;

19 class = 'EDSS 2';

20 class = 'EDSS 3';

21 if $\mathrm{L}<1536.59$ then node 28 else node 29;

22 class = 'EDSS 4';

23 class = 'EDSS 3';

24 if TTV $<6.04$ then node 30 else node 31;

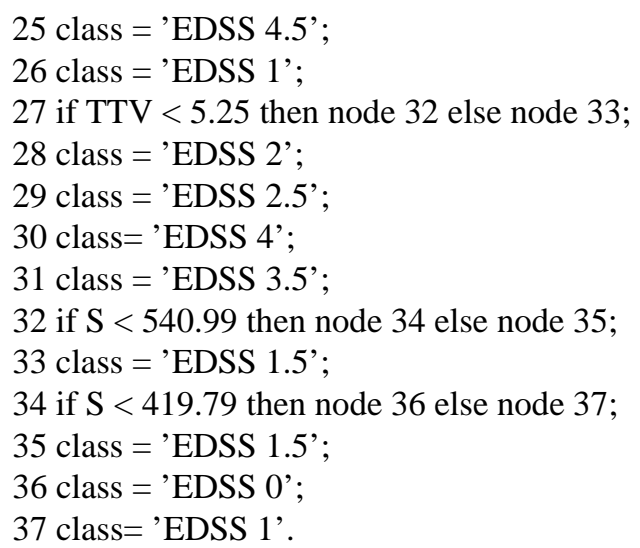

Table 2 showed \%Agreement obtained by comparing clinical EDSS scores of the test group and ones estimated using each model. The best agreement (75\%) with the clinical EDSS scores was obtained by the decision tree. The distribution of the related errors (error = estimated score - clinical score) was presented in the Figure 2. The absolute of the error for the disagreement portion (25\%) was $1.6 \pm 0.5$ EDSS.

\section{Discussion}

In this study, one performance decision tree was constructed with five measures, including two linear measures ( $\mathrm{S}$ and $\mathrm{L}$ ) and three non-linear measures (RecP, TTV and TTA), extracted from posturologic data, because it revealed a better agreement with clinical scores

Table 2. \%Agreement for the test group by using decision tree and polynomial regression models.

\begin{tabular}{lcc}
\hline \multicolumn{1}{c}{ Model } & Measure & \%Agreement \\
\hline Decision tree & S, L, RecP, TTV, TTA & $75 \%$ \\
$\begin{array}{lcc}\text { Second-order polynomial } \\
\text { regression models }\end{array}$ & S & $35 \%$ \\
& RecP & $45 \%$ \\
\hline
\end{tabular}

\%Agreement: pencentage of agreement; S: surface of the ellipse; L: total length of the COP path; RecP: recurrence rate of COP's position; TTV: trapping time of COP's velocity; TTA: trapping time of COP's acceleration.

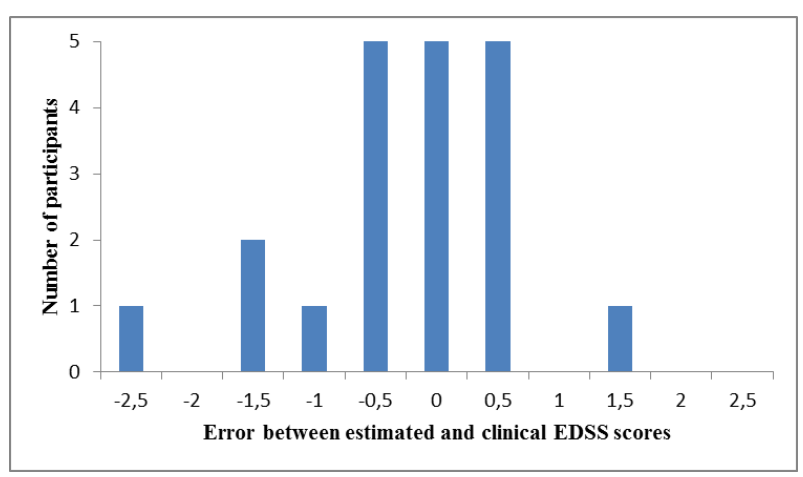

Figure 2. Error between estimated and clinical EDSS scores of the test group by using the selected decision tree. 
of the test group than two polynomial regression models (S and RecP) which had shown very good agreement with ones of the learning group [6]. That may be caused by using a combination of several measures in this model and the combination can reduce the error generated by using only one measure. Meanwhile, we introduced our tree two non-linear measures (TTV and TTA) which are able to evaluate the complexity of a dynamic system, because TT represents the average time in which the system is trapped in a specific state [9].

In our selected decision tree, the root node (in the level 1 ) is S (node 1) and the nodes in the level 2 are S (node 3) and TTA (node 2). That means S and TTA are two dominant measures in the classification for EDSS scores. Just as we expected, the patients could be directly classified into three principal groups using S: if S > 2149.38 $\mathrm{mm}^{2}$, EDSS score is between 3 and 4.5 (high scores); if $910.53 \mathrm{~mm}^{2}<\mathrm{S}<2149.38 \mathrm{~mm}^{2}$, EDSS is between 2 and 3 (medium scores); if $\mathrm{S}<910.53 \mathrm{~mm}^{2}$, EDSS is between 0 and 3 (low-medium scores). For the group of low-medium EDSS score, we can continue classifying them by using TTA: if TTA < 15, EDSS ranges from 2 to 3 (medium scores); otherwise, EDSS ranges from 0 to 2 (low scores). From that, we have observed that $\mathrm{S}$ (linear measure) allows selecting the patients with high and medium EDSS scores, while TTA (non-linear measure) allows selecting low EDSS scores. Generally, patients with high EDSS scores have a great $\mathrm{S}$ [3]. Thus, $\mathrm{S}$ is the most important measure to classify EDSS, especially to identify high scores. However, it is not sufficient to distinguish all the scores. To identify low scores, we need to take into account TTA. When TTA is high, the COP's instantaneous acceleration is trapped for much amount of time, and its oscillation is small around an equilibrium position (such as EDSS between 0 and 2). On the contrary, TTA becomes lower as the variation of the acceleration increases (such as EDSS between 2 and 3), because the increase of the variation is becoming complex and system dynamics are changing fast due to its faster COP's displacement over the time. As TTA plays an important role to identify low EDSS scores between 0 and 2, it may be a good indicator linked with $\mathrm{S}$ to predict the emergence of MS. In addition of these two measures, L, RecP and TTV should be considered to assess EDSS scores within each principal group.

\section{Conclusion}

In this paper, we presented a method for assessing EDSS score from postural data of patients with MS using a decision tree with five measures (S, L, RecP, TTV and TTA). The results have signified that our tree model with a combination of some measures is able to automatically assess the EDSS scores and that it is possible to distinguish the EDSS scores by using linear and non-linear postural sway measures. It would be interesting to test other values of the time delay and the embedding dimension for RQA and to study the difference between the sexes in the future research.

\section{Acknowledgements}

The authors would like to thank the Hôpital de SaintPhilibert, Lomme, for providing posturologic data of the healthy subjects and the patients with multiple sclerosis.

\section{REFERENCES}

[1] J. F. Kurtzke, "Rating Neurologic Impairment in Multiple Sclerosis: An Expanded Disability Status Scale (EDSS)," Neurology, Vol. 33, No. 11, 1983, pp. 1444-1452.

http://dx.doi.org/10.1212/WNL.33.11.1444

[2] B. Sharrack and R. A. Hughes, "Clinical Scales for Multiple Sclerosis," Journal of the Neurological Sciences, Vol. 135, No. 1, 1996, pp. 1-9.

http://dx.doi.org/10.1016/0022-510X(95)00261-Y

[3] A. Yahia, S. Ghroubi, C. Mhiri and M. H. Elleuch, "Relationship between Muscular Strength, Gait and Postural Parameters in Multiple Sclerosis,” Annals of Physical and Rehabilitation Medicine, Vol. 54, No. 3, 2011, pp. 144155. http://dx.doi.org/10.1016/j.rehab.2011.02.004

[4] A. Porosinska, K. Pierzchala, M. Mentel and J. Karpe, "Evaluation of Postural Balance Control in Patients with Multiple Sclerosis-Effect of Different Sensory Conditions and Arithmetic Task Execution. A Pilot Study,” Neurologia i Neurochirurgia Polskae, Vol. 44, No. 1, 2010, pp. 35-42.

[5] L. Peyrodie, S. Boudet, A. Pinti, F. Cavillon, O. Agnani and P. Gallois, "Relations Entre Posturologie et Score EDSS," Sciences et Technologies pour le Handicap, Vol. 4, No. 1, 2010, pp. 55-71.

http://dx.doi.org/10.3166/sth.4.55-71

[6] H. Cao, L. Peyrodie, S. Boudet, F. Cavillon, C. Donzé and O. Agnani, "Estimation of Expanded Disability Status Scale (EDSS) from Posturographic Data in Multiple Sclerosis," Unpublished.

[7] N. Marwan, M. Carmenromano, M. Thiel and J. Kurths, "Recurrence Plots for the Analysis of Complex Systems," Physics Reports, Vol. 438, No. 5-6, 2007, pp. 237-329. http://dx.doi.org/10.1016/j.physrep.2006.11.001

[8] R. O. Duda, P. E. Hart and D. G. Stork, "Pattern Classification,” Wiley, New York, 2001.

[9] S. D. Mhalsekar, S. S. Rao and K. V. Gangadharan, "Investigation on Feasibility of Recurrence Quantification Analysis for Detecting Flank Wear in Face Milling," International Journal of Engineering, Science and Technology, Vol. 2, No. 5, 2010, pp. 23-38. 